${ }^{10}$ Pauli, W., Theory of Relativity, 151-152 (Pergamon, New York, 1958).

11 Pathria, R. K., Theory of Relativity (Pergamon, Oxford, 1974).

12 Price, H. E., Am. J. Phys., 42, 336 (1974).

\section{Gravitational searchlight and its astrophysical applications}

WE wish to point out an interesting effect associated with light emitted in the forward direction by a source circulating in an equatorial plane around a highly collapsed object or a black hole of mass $M$. The essential feature of the emission is that provided the radius $a$ of the orbit in Schwarzschild's coordinates exceeds $3 G M / c^{2}$, the light emitted is blueshifted when received by a distant observer. More specifically, if $v_{0}$ is the frequency of light at emission and $v$ the frequency at reception at a radial coordinate $R \gg R_{\mathrm{s}}=2 G M / c^{2}$, we have

$$
\frac{v}{v_{0}}=\frac{\sqrt{ }[(2-3 \xi)(1-\xi)]}{\sqrt{ }[2(1-\xi)]-\sqrt{ } \xi}
$$

where

$$
\xi=\mathbf{R}_{\mathbf{s}} / a
$$

While $v>v_{0}$ for all values of $\xi<2 / 3$, it can be shown easily that as $\xi \rightarrow 2 / 3$, the blueshift diverges. Writing $\xi=(2 / 3)(1-\varepsilon)$,

$$
v \simeq(2 / 3) v_{0} \varepsilon^{-1 / 2} \text { as } \varepsilon \rightarrow 0
$$

Thus very high blueshifts are obtained as the orbit of the particle tends to the so called unstable circular orbit. Qualitatively, we can understand the result in terms of the competition between the Doppler blueshift of forward light emission and the gravitational redshift due to the central mass. The former increases much more rapidly than the latter as $\varepsilon \rightarrow 0$. We are interested here with orbits with very small $\varepsilon$, that is, orbits close to the unstable circular orbit.

The behaviour of matter revolving very close to such an orbit has been considered by several authors ${ }^{1-3}$. For example, an electron moving in a circular orbit can emit synchrotrontype radiation. It is also possible for gravitational radiation to be emitted by revolving matter. Such effects, however, have turned out to be very small even in the limiting case of $\varepsilon \rightarrow 0$. Here we suggest an alternative situation where the above mentioned result can play a significant part.

Before coming to the astrophysical setting consider what happens when we have matter moving in a thin ring round an object with mass $M$, with say $\varepsilon_{1} \leqslant \varepsilon \leqslant \varepsilon_{2} \leqslant 1$. Suppose matter in this region has uniform distribution and is emitting light in a band of frequencies peaked round $v_{0}$, say. Then purely geometrical considerations and equation (3) lead to a reception spectrum at $R$ of the form

$$
F(v)=A\left(v_{0}\right) v^{-1}, v \geqslant v_{0}
$$

The function $A\left(v_{0}\right)$ depends on the dimensions of the object and the emissivity of matter under consideration. The derivation of equations (3) and (4) is somewhat involved and will be published elsewhere, but the $v^{-1}$ dependence of the spectrum purely on geometrical ground is noteworthy.

Turning now to the astrophysical considerations, it is well known that a highly collapsed object or a black hole tends to accrete matter. In general the infalling matter has some angular momentum so that it does not fall in radially. Instead, one can imagine matter making revolutions round the object several times before falling in. The unstable circular orbit is likely to play an interesting part in this process. The infalling matter goes round in orbits with small $\varepsilon$ several times before getting sucked in or thrown out again'. So we may consider the thin ring discussed above as made up of transient matter.

The $v^{-1}$ spectrum is common to many extragalactic sources of radiation. We have considered the continuum emission of QSOs whose spectrum can be approximated this way ${ }^{4}$. By considering thermal emission from hot gas in a narrow range of frequencies around $v_{0} \sim 3 \times 10^{14} \mathrm{~Hz}$ and taking the emissivity as $\sim 4.4 \times 10^{-24} n_{\mathrm{e}}{ }^{2} \mathrm{erg} \mathrm{cm}^{-3} \mathrm{~s}^{-1}$, with $n_{\mathrm{e}}$ the electron density in $\mathrm{cm}^{-3}$ we find that in order to explain the observations of a QSO like 3C273 in the optical and ultraviolet region on the basis of equation (4) we need a mass-distance relationship of the form

$$
M / M_{\odot} \simeq 1.7 \times 10^{15}\left(R_{\mathrm{mpc}} / n_{\mathrm{e}}\right)^{2 / 3}
$$

Here $R_{\mathrm{mpc}}$ is $R$ expressed in Mpc. In a 'local hypothesis', $R_{\text {mpc }}$ may not exceed $\sim 100$ while $n_{\mathrm{c}}$ can be taken as high as $\sim 10^{\ominus}$ to give a mass $M$ of the order of that of a galaxy. Provided thermal emission is confined to relatively narrow band of frequencies at the source, the resulting spectrum at a large distance will show a $v^{-1}$ dependence.

The light emitted in the forward direction makes several rounds of the object before reaching the distant receiver. As $\varepsilon \rightarrow 0$, the angle through which the light has turned can be shown to be

$$
\varphi \sim \ln [(24-12 \sqrt{ } 3) / \varepsilon]
$$

Thus for $\varepsilon \sim 10^{-10}$ the light will go round nearly four times.

J. V. N. thanks the Jawaharlal Nehru Fund for the award of a Fellowship. R. C. K. thanks the Tata Institute of Fundamental Research for hospitality.

S. M. ChITRE

J. V. NARLIKAR

Tata Institute of Fundamental Research, Bombay 400005

Indian Institute of Astrophysics, Bangalore 560 006, India

Received September 9, 1974.

1 Misner, C. W., Thorne, K. S., and Wheeler, J. A., Gravitation, chapter 25 (Freeman, San Francisco, 1973).

${ }^{2}$ Misner, C. W., Breuer, R. A., Brill, D. R., Chrzanowski, P. L., Hughes, H. G., III, and Pereira, C. M., Phys. Rev. Lett., 28, 998 (1972).

3 Hughes, H. G., III, Ann. Phys., 80, 463 (1973).

4 Burbidge, G. R., and Burbidge, E. M., Quasistellar objects, chapter 4 (Freeman, San Francisco, 1967).

${ }^{5}$ Silk, J., Astrophys. J., 160, 793 (1970).

\section{Solar energetic particle event with $3 \mathrm{He} / 4 \mathbf{H e}>1$}

OBSERVATIONS of $\gamma$ rays and the short lived isotope ${ }^{3} \mathrm{H}$ provide evidence for the occurrence of nuclear reactions by high energy particles accelerated in solar flares ${ }^{1,2}$. Stable isotopes such as ${ }^{2} \mathrm{H}$ and ${ }^{3} \mathrm{He}$ should also be produced in these reactions. Solar ${ }^{3} \mathrm{He}$ particles were first detected by $\mathrm{Hsieh}$ and $\mathrm{Simpson}^{3}$. In the energy range 10 to $100 \mathrm{MeV}$ per nucleon they obtained ${ }^{3} \mathrm{He} /{ }^{4} \mathrm{He}=(2.1 \pm 0.4) \times 10^{-2}$ by summing over seven solar particle events. Garrard et al. ${ }^{4}$ and Anglin et al..$^{5}$ have reported that ${ }^{3} \mathrm{He} /{ }^{4} \mathrm{He}$ was highly variable from event to event. (Table 1 ). In the "${ }^{3} \mathrm{He}$-rich events', ${ }^{2} \mathrm{H}$ and ${ }^{3} \mathrm{H}$ were not detected and the resulting upper limits were much less than expected from the theory of nuclear reactions ${ }^{6}$. These events were small, and the 